\title{
A strong convergence theorem for equilibrium problems and split feasibility problems in Hilbert spaces
}

Jinfang Tang ${ }^{1}$, Shih-sen Chang ${ }^{2 *}$ and Fei Yuan ${ }^{3}$

\section{"Correspondence:}

changss2013@aliyun.com

${ }^{2}$ College of Statistics and

Mathematics, Yunnan University of

Finance and Economics, Kunming, Yunnan 650221, China

Full list of author information is

available at the end of the article

\begin{abstract}
The main purpose of this paper is to introduce an iterative algorithm for equilibrium problems and split feasibility problems in Hilbert spaces. Under suitable conditions we prove that the sequence converges strongly to a common element of the set of solutions of equilibrium problems and the set of solutions of split feasibility problems. Our result extends and improves the corresponding results of some others.
\end{abstract}

MSC: $90 \mathrm{C} 25 ;$ 90C30; 47J25; 47H09

Keywords: equilibrium problems; split feasibility problems; strong convergence; bounded linear operator; fixed point

\section{Introduction}

Let $H$ be a real Hilbert space with inner product $\langle\cdot, \cdot\rangle$ and induced norm $\|\cdot\|$, let $F: H \times$ $H \rightarrow \mathbb{R}$ be a bifunction. Then we consider the following equilibrium problem (EP): find $z \in H$ such that

$$
F(z, y) \geq 0, \quad \forall y \in H
$$

The set of the EP is denoted by $\Omega$, i.e.,

$$
\Omega=\{z \in H: F(z, y) \geq 0, \forall y \in H\} .
$$

The problem (1.1) is very general in the sense that it includes, as special cases, optimization problems, variational inequality problems, the Nash equilibrium problems and others, see, for instance, [1-3]. Some methods have been proposed to solve the EP, see, e.g., [4-6] and $[7,8]$.

The split feasibility problem (SFP) was proposed by Censer and Elfving in [9]. It can be formulated as the problem of finding a point $x$ satisfying the property:

$$
x \in C, \quad A x \in Q,
$$

where $A$ is a given $M \times N$ real matrix, and $C$ and $Q$ are nonempty, closed and convex subsets in $\mathbb{R}^{N}$ and $\mathbb{R}^{M}$, respectively.

@2014 Tang et al.; licensee Springer. This is an Open Access article distributed under the terms of the Creative Commons Attribution License (http://creativecommons.org/licenses/by/2.0), which permits unrestricted use, distribution, and reproduction in any medium, provided the original work is properly cited. 
Due to its extraordinary utility and broad applicability in many areas of applied mathematics (most notably, fully discretized models of problems in image reconstruction from projections, in image processing, and in intensity-modulated radiation therapy), algorithms for solving convex feasibility problems have been received great attention (see, for instance [10-13] and also [14-18]).

We assume the SFP (1.2) is consistent, and let $\Gamma$ be the solution set, i.e.,

$$
\Gamma=\{x \in C: A x \in Q\}
$$

It is not hard to see that $\Gamma$ is closed convex and $x \in \Gamma$ if and only if it solves the fixed-point equation

$$
x=P_{C}\left(I-\gamma A^{*}\left(I-P_{Q}\right) A\right) x,
$$

where $P_{C}$ and $P_{Q}$ are the orthogonal projection onto $C$ and $Q$, respectively, $\gamma>0$ is any positive constant and $A^{*}$ denotes the adjoint of $A$.

Recently, for the purpose of generality, the SFP (1.2) has been studied in a more general setting. For instance, see $[16,19]$. However, the algorithms in these references have only weak convergence in the setting of infinite-dimensional Hilbert spaces. Very recently, $\mathrm{He}$ and Zhao [20] introduce a new relaxed CQ algorithm (1.4) such that the strong convergence is guaranteed in infinite-dimensional Hilbert spaces:

$$
x_{n+1}=P_{C_{n}}\left(\alpha_{n} u+\left(1-\alpha_{n}\right)\left(x_{n}-\tau_{n} \nabla f_{n}\left(x_{n}\right)\right)\right) \text {. }
$$

Motivated and inspired by the research going on in the sections of equilibrium problems and split feasibility problems, the purpose of this article is to introduce an iterative algorithm for equilibrium problems and split feasibility problems in Hilbert spaces. Under suitable conditions we prove the sequence converges strongly to a common element of the set of solutions of equilibrium problems and the set of solutions of split feasibility problems. Our result extends and improves the corresponding results of He et al. [20] and some others.

\section{Preliminaries and lemmas}

Throughout this paper, we assume that $H, H_{1}$ or $H_{2}$ is a real Hilbert space, $A$ is a bounded linear operator from $H_{1}$ to $H_{2}$, and $I$ is the identity operator on $H, H_{1}$ or $H_{2}$. If $f: H \rightarrow \mathbb{R}$ is a differentiable function, then we denote by $\nabla f$ the gradient of the function $f$. We will also use the notations: $\rightarrow$ to denote strong convergence, $\rightarrow$ to denote weak convergence and to denote by

$$
w_{\omega}\left(x_{n}\right)=\left\{x \mid \exists\left\{x_{n_{k}}\right\} \subset\left\{x_{n}\right\} \text { such that } x_{n_{k}} \rightarrow x\right\}
$$

the weak $\omega$-limit set of $\left\{x_{n}\right\}$.

Recall that a mapping $T: H \rightarrow H$ is said to be nonexpansive if

$$
\|T x-T y\| \leq\|x-y\|, \quad x, y \in H .
$$


$T: H \rightarrow H$ is said to be firmly nonexpansive if

$$
\|T x-T y\|^{2} \leq\|x-y\|^{2}-\|(I-T) x-(I-T) y\|^{2}, \quad x, y \in H .
$$

A mapping $T: H \rightarrow H$ is said to be demi-closed at origin, if for any sequence $\left\{x_{n}\right\} \subset H$ with $x_{n} \rightarrow x^{*}$ and $\lim _{n \rightarrow \infty}\left\|(I-T) x_{n}\right\|=0$, then $x^{*}=T x^{*}$.

It is easy to prove that if $T: H \rightarrow H$ is a firmly nonexpansive mapping, then $T$ is demiclosed at the origin.

A function $f: H \rightarrow \mathbb{R}$ is called convex if

$$
f(\lambda x+(1-\lambda) y) \leq \lambda f(x)+(1-\lambda) f(y), \quad \forall \lambda \in(0,1), \forall x, y \in H .
$$

Lemma 2.1 Let $T: \mathrm{H}_{2} \rightarrow \mathrm{H}_{2}$ be a firmly nonexpansive mapping such that $\|(I-T) x\|$ is a convex function from $H_{2}$ to $\overline{\mathbb{R}}=[-\infty,+\infty]$. Let $A: H_{1} \rightarrow H_{2}$ be a bounded linear operator and

$$
f(x):=\frac{1}{2}\|(I-T) A x\|^{2}, \quad \forall x \in H_{1} .
$$

Then

(i) $\nabla f(x)=A^{*}(I-T) A x, x \in H_{1}$.

(ii) $\nabla f$ is $\|A\|^{2}$-Lipschitz, i.e., $\|\nabla f(x)-\nabla f(y)\| \leq\|A\|^{2}\|x-y\|, x, y \in H_{1}$.

Proof (i) From the definition of $f$, we know that $f$ is convex. First we prove that the limit

$$
\langle\nabla f(x), v\rangle=\lim _{h \rightarrow 0+} \frac{f(x+h v)-f(x)}{h}
$$

exists in $\overline{\mathcal{R}}:=\{-\infty\} \cup \mathbb{R} \cup\{+\infty\}$ and satisfies

$$
\langle\nabla f(x), v\rangle \leq f(x+v)-f(x), \quad \forall v \in H_{1} .
$$

If fact, if $0<h_{1} \leq h_{2}$, then

$$
f\left(x+h_{1} v\right)-f(x)=f\left(\frac{h_{1}}{h_{2}}\left(x+h_{2} v\right)+\left(1-\frac{h_{1}}{h_{2}}\right) x\right)-f(x) .
$$

Since $f$ is convex and $\frac{h_{1}}{h_{2}} \leq 1$, it follows that

$$
f\left(x+h_{1} v\right)-f(x) \leq \frac{h_{1}}{h_{2}} f\left(x+h_{2} v\right)+\left(1-\frac{h_{1}}{h_{2}}\right) f(x)-f(x),
$$

and hence that

$$
\frac{f\left(x+h_{1} v\right)-f(x)}{h_{1}} \leq \frac{f\left(x+h_{2} v\right)-f(x)}{h_{2}} .
$$

This shows that this difference quotient is increasing, therefore it has a limit in $\overline{\mathcal{R}}$ as $h \rightarrow 0+:$

$$
\langle\nabla f(x), v\rangle=\inf _{h>0} \frac{f(x+h v)-f(x)}{h}=\lim _{h \rightarrow 0+} \frac{f(x+h v)-f(x)}{h} .
$$


This implies that $f$ is differential. Taking $h=1,(2.1)$ implies that

$$
\langle\nabla f(x), v\rangle \leq f(x+v)-f(x)
$$

Next we prove that

$$
\nabla f(x)=A^{*}(I-T) A x, \quad x \in H_{1} .
$$

In fact, since

$$
\lim _{h \rightarrow 0+} \frac{f(x+h v)-f(x)}{h}=\lim _{h \rightarrow 0+} \frac{\|A x+h A v-T A(x+h v)\|^{2}-\|(I-T) A x\|^{2}}{2 h}
$$

and

$$
\begin{aligned}
\| A x & +h A v-T A(x+h v)\left\|^{2}-\right\|(I-T) A x \|^{2} \\
= & \|A x\|^{2}+h^{2}\|A v\|^{2}+2 h\left\langle A^{*} A x, v\right\rangle+\|T A(x+h v)\|^{2}-\|A x\|^{2}-\|T A x\|^{2} \\
& -2\langle A x, T A(x+h v)-T A x\rangle-2 h\left\langle A^{*} T A(x+h v), v\right\rangle .
\end{aligned}
$$

Substituting (2.3) into (2.2), simplifying and then letting $h \rightarrow 0+$ and taking the limit we have

$$
\begin{aligned}
\lim _{h \rightarrow 0+} \frac{f(x+h v)-f(x)}{h} & =\lim _{h \rightarrow 0+} \frac{2 h\left\{\left\langle A^{*} A x, v\right\rangle-\left\langle A^{*} T A(x+h v), v\right\rangle\right\}}{2 h} \\
& =\left\langle A^{*}(I-T) A x, v\right\rangle, \quad \forall v \in H_{1} .
\end{aligned}
$$

It follows from (2.1) that

$$
\nabla f(x)=A^{*}(I-T) A x, \quad x \in H_{1} .
$$

(ii) From (i) we have

$$
\begin{aligned}
\|\nabla f(x)-\nabla f(y)\| & =\left\|A^{*}(I-T) A x-A^{*}(I-T) A y\right\| \\
& =\left\|A^{*}[(I-T) A x-(I-T) A y]\right\| \\
& \leq\|A\|\|A x-A y\| \leq\|A\|^{2}\|x-y\|, \quad x, y \in H_{1} .
\end{aligned}
$$

Lemma 2.2 (See, for example, [21]) Let $T: H \rightarrow H$ be an operator. The following statements are equivalent.

(i) $T$ is firmly nonexpansive.

(ii) $\|T x-T y\|^{2} \leq\langle x-y, T x-T y\rangle, \forall x, y \in H$.

(iii) $I-T$ is firmly nonexpansive.

Proof (i) $\Rightarrow$ (ii): Since $T$ is firmly nonexpansive, for all $x, y \in H$ we have

$$
\begin{aligned}
\|T x-T y\|^{2} & \leq\|x-y\|^{2}-\|(I-T) x-(I-T) y\|^{2} \\
& =\|x-y\|^{2}-\|x-y\|^{2}-\|T x-T y\|^{2}+2\langle x-y, T x-T y\rangle \\
& =2\langle x-y, T x-T y\rangle-\|T x-T y\|^{2},
\end{aligned}
$$


hence

$$
\|T x-T y\|^{2} \leq\langle x-y, T x-T y\rangle, \quad \forall x, y \in H .
$$

(ii) $\Rightarrow$ (iii): From (ii), we know that for all $x, y \in H$

$$
\begin{aligned}
\|(I-T) x-(I-T) y\|^{2} & =\|(x-y)-(T x-T y)\|^{2} \\
& =\|x-y\|^{2}-2\langle x-y, T x-T y\rangle+\|T x-T y\|^{2} \\
& \leq\|x-y\|^{2}-\langle x-y, T x-T y\rangle \\
& =\langle x-y,(I-T) x-(I-T) y\rangle .
\end{aligned}
$$

This implies that $I-T$ is firmly nonexpansive.

(iii) $\Rightarrow$ (i): From (iii) we immediately know that $T$ is firmly nonexpansive.

Let $C$ be a nonempty closed convex subset of $H$. Recall that for every point $x \in H$, there exists a unique nearest point of $C$, denoted by $P_{C} x$, such that $\left\|x-P_{C} x\right\| \leq\|x-y\|$ for all $y \in C$. Such a $P_{C}$ is called the metric projection from $H$ onto $C$. We know that $P_{C}$ is a firmly nonexpansive mapping from $H$ onto $C$, i.e.,

$$
\left\|P_{C} x-P_{C} y\right\|^{2} \leq\left\langle P_{C} x-P_{C} y, x-y\right\rangle, \quad \forall x, y \in H .
$$

Further, for any $x \in H$ and $z \in C, z=P_{C} x$ if and only if

$$
\langle x-z, z-y\rangle \geq 0, \quad \forall y \in C .
$$

Throughout this paper, let us assume that a bifunction $F: H \times H \rightarrow \mathbb{R}$ satisfies the following conditions:

(A1) $F(x, x)=0, \forall x \in H$;

(A2) $F$ is monotone, i.e., $F(x, y)+F(y, x) \leq 0, \forall x, y \in H$;

(A3) $\lim _{t \downarrow 0} F(t z+(1-t) x, y) \leq F(x, y), \forall x, y, z \in H$;

(A4) for each $x \in H, y \mapsto F(x, y)$ is convex and lower semicontinuous.

Lemma $2.3([1,4])$ Let $H$ be a Hilbert space and let $F: H \times H \rightarrow \mathbb{R}$ satisfy (A1), (A2), (A3), and (A4). Then, for any $r>0$ and $x \in H$, there exists $z \in H$ such that

$$
F(z, y)+\frac{1}{r}\langle y-z, z-x\rangle \geq 0, \quad \forall y \in H .
$$

Furthermore, if

$$
T_{r} x=\left\{z \in H: F(z, y)+\frac{1}{r}\langle y-z, z-x\rangle \geq 0, \forall y \in H\right\},
$$

then the following hold:

(1) $T_{r}$ is single-valued;

(2) $T_{r}$ is firmly nonexpansive;

(3) $F\left(T_{r}\right)=\Omega$;

(4) $\Omega$ is closed and convex. 
The following results play an important role in this paper.

Lemma 2.4 ([22]) Let X be a real Hilbert space, then we have

$$
\|x+y\|^{2} \leq\|x\|^{2}+2\langle y, x+y\rangle, \quad \forall x, y \in X .
$$

Lemma 2.5 ([23]) Let $\left\{x_{n}\right\}$ and $\left\{y_{n}\right\}$ be bounded sequences in a Banach space X. Let $\left\{\beta_{n}\right\}$ be a sequence in $[0,1]$ satisfying $0<\liminf _{n \rightarrow \infty} \beta_{n} \leq \limsup _{n \rightarrow \infty} \beta_{n}<1$. Suppose that

$$
x_{n+1}=\left(1-\beta_{n}\right) y_{n}+\beta_{n} x_{n}
$$

for all integer $n \geq 0$ and

$$
\limsup _{n \rightarrow \infty}\left(\left\|y_{n+1}-y_{n}\right\|-\left\|x_{n+1}-x_{n}\right\|\right) \leq 0
$$

Then $\lim _{n \rightarrow \infty}\left\|y_{n}-x_{n}\right\|=0$.

Lemma 2.6 ([24]) Let $\left\{a_{n}\right\}$ be a sequence of nonnegative real numbers such that

$$
a_{n+1} \leq\left(1-\gamma_{n}\right) a_{n}+\gamma_{n} \sigma_{n}, \quad n=0,1,2, \ldots
$$

where $\left\{\gamma_{n}\right\}$ is a sequence in $(0,1)$, and $\left\{\sigma_{n}\right\}$ is a sequence in $\mathbb{R}$ such that

(i) $\sum_{n=0}^{\infty} \gamma_{n}=\infty$;

(ii) $\lim \sup _{n \rightarrow \infty} \sigma_{n} \leq 0$, or $\sum_{n=0}^{\infty}\left|\gamma_{n} \sigma_{n}\right|<\infty$.

Then $\lim _{n \rightarrow \infty} a_{n}=0$.

\section{Main results}

We are now in a position to prove the following theorem.

Theorem 3.1 Let $H_{1}, H_{2}$ be two real Hilbert spaces, $F: H_{1} \times H_{1} \rightarrow \mathbb{R}$ be a bifunction satisfying (A1), (A2), (A3), and (A4). Let $A: H_{1} \rightarrow H_{2}$ be a bounded linear operator, $S: H_{1} \rightarrow H_{1}$ be a firmly nonexpansive mapping, and let $T: H_{2} \rightarrow H_{2}$ be a firmly nonexpansive mapping such that $\|(I-T) x\|$ is a convex function from $H_{2}$ to $\mathbb{R}$. Assume that $C:=F(S) \cap \Omega \neq \emptyset$ and $Q:=F(T) \neq \emptyset$. Let $u \in H_{1}$ and $\left\{x_{n}\right\}$ be the sequence generated by

$$
\left\{\begin{array}{l}
x_{0} \in H_{1} \text { chosen arbitrarily, } \\
x_{n+1}=\beta_{n} x_{n}+\left(1-\beta_{n}\right) y_{n}, \\
F\left(y_{n}, x\right)+\frac{1}{\lambda_{n}}\left\langle x-y_{n}, y_{n}-z_{n}\right\rangle \geq 0, \quad \forall x \in H_{1}, \\
z_{n}=S\left(\alpha_{n} u+\left(1-\alpha_{n}\right)\left(x_{n}-\xi_{n} \nabla f\left(x_{n}\right)\right)\right),
\end{array}\right.
$$

where

$$
\begin{aligned}
& f\left(x_{n}\right)=\frac{1}{2}\left\|(I-T) A x_{n}\right\|^{2}, \quad \nabla f\left(x_{n}\right)=A^{*}(I-T) A x_{n} \neq 0 \quad \forall n \geq 1, \\
& \xi_{n}=\frac{\rho_{n} f\left(x_{n}\right)}{\left\|\nabla f\left(x_{n}\right)\right\|^{2}} .
\end{aligned}
$$


If the solution set $\Gamma$ of $\operatorname{SPF}(1.2)$ is not empty, and the sequences $\left\{\rho_{n}\right\} \subset(0,4),\left\{\alpha_{n}\right\},\left\{\beta_{n}\right\} \subset$ $(0,1)$ satisfy the following conditions:

(i) $\lim _{n \rightarrow \infty} \alpha_{n}=0, \sum_{n=0}^{\infty} \alpha_{n}=\infty$;

(ii) $0<\liminf _{n \rightarrow \infty} \beta_{n} \leq \limsup _{n \rightarrow \infty} \beta_{n}<1$;

(iii) $\lambda_{n} \in(a, b) \subset(0,+\infty)$ and $\lim _{n \rightarrow \infty}\left(\lambda_{n+1}-\lambda_{n}\right)=0$,

then the sequence $\left\{x_{n}\right\}$ converges strongly to $P_{\Gamma} u$.

Proof Since the solution set $\Omega$ of EP and the solution set of SPF (1.2) are both closed and convex, $\Gamma(\neq \emptyset)$ is closed and convex. Thus, the metric projection $P_{\Gamma}$ is well defined.

Letting $p=P_{\Gamma} u$, it follows from Lemma 2.3 that $y_{n}=T_{\lambda_{n}} z_{n}$ and

$$
\left\|y_{n}-p\right\|=\left\|T_{\lambda_{n}} z_{n}-T_{\lambda_{n}} p\right\| \leq\left\|z_{n}-p\right\| .
$$

Observing that $S$ is firmly nonexpansive, we have

$$
\begin{aligned}
\left\|z_{n}-p\right\| & =\left\|S\left(\alpha_{n} u+\left(1-\alpha_{n}\right)\left(x_{n}-\xi_{n} \nabla f\left(x_{n}\right)\right)\right)-p\right\| \\
& \leq\left\|\alpha_{n}(u-p)+\left(1-\alpha_{n}\right)\left(x_{n}-\xi_{n} \nabla f\left(x_{n}\right)-p\right)\right\| \\
& \leq \alpha_{n}\|u-p\|+\left(1-\alpha_{n}\right)\left\|x_{n}-\xi_{n} \nabla f\left(x_{n}\right)-p\right\| .
\end{aligned}
$$

Since $p \in \Gamma \subset C, \nabla f(p)=0$. Observe that $I-T$ is firmly nonexpansive, from Lemma 2.2(ii) we have

$$
\begin{aligned}
\left\langle\nabla f\left(x_{n}\right), x_{n}-p\right\rangle & =\left\langle(I-T) A x_{n}, A x_{n}-A p\right\rangle \\
& \geq\left\|(I-T) A x_{n}\right\|^{2}=2 f\left(x_{n}\right) .
\end{aligned}
$$

This implies that

$$
\begin{aligned}
\left\|x_{n}-\xi_{n} \nabla f\left(x_{n}\right)-p\right\|^{2} & =\left\|x_{n}-p\right\|^{2}+\left\|\xi_{n} \nabla f\left(x_{n}\right)\right\|^{2}-2 \xi_{n}\left\langle\nabla f\left(x_{n}\right), x_{n}-p\right\rangle \\
& \leq\left\|x_{n}-p\right\|^{2}+\xi_{n}^{2}\left\|\nabla f\left(x_{n}\right)\right\|^{2}-4 \xi_{n} f\left(x_{n}\right) \\
& =\left\|x_{n}-p\right\|^{2}-\rho_{n}\left(4-\rho_{n}\right) \frac{f^{2}\left(x_{n}\right)}{\left\|\nabla f\left(x_{n}\right)\right\|^{2}} \\
& \leq\left\|x_{n}-p\right\|^{2} .
\end{aligned}
$$

Substituting (3.5) into (3.3), we get

$$
\left\|z_{n}-p\right\| \leq \alpha_{n}\|u-p\|+\left(1-\alpha_{n}\right)\left\|x_{n}-p\right\| .
$$

Thus, from (3.2) and (3.6) we have

$$
\begin{aligned}
\left\|x_{n+1}-p\right\| & =\left\|\beta_{n} x_{n}+\left(1-\beta_{n}\right) y_{n}-p\right\| \\
& \leq \beta_{n}\left\|x_{n}-p\right\|+\left(1-\beta_{n}\right)\left\|y_{n}-p\right\| \\
& \leq \beta_{n}\left\|x_{n}-p\right\|+\left(1-\beta_{n}\right)\left\|z_{n}-p\right\| \\
& \leq\left(1-\alpha_{n}\left(1-\beta_{n}\right)\right)\left\|x_{n}-p\right\|+\alpha_{n}\left(1-\beta_{n}\right)\|u-p\| .
\end{aligned}
$$


It turns out that

$$
\left\|x_{n+1}-p\right\| \leq \max \left\{\left\|x_{n}-p\right\|,\|u-p\|\right\} .
$$

By induction, we have

$$
\left\|x_{n}-p\right\| \leq \max \left\{\left\|x_{0}-p\right\|,\|u-p\|\right\} .
$$

This implies that the sequence $\left\{x_{n}\right\}$ is bounded. From (3.2) and (3.6) we know that $\left\{y_{n}\right\}$ and $\left\{z_{n}\right\}$ both are bounded.

From Lemma 2.4 and (3.5), we have

$$
\begin{aligned}
\left\|z_{n}-p\right\|^{2}= & \left\|S\left(\alpha_{n} u+\left(1-\alpha_{n}\right)\left(x_{n}-\xi_{n} \nabla f\left(x_{n}\right)\right)\right)-p\right\|^{2} \\
\leq & \left\|\alpha_{n}(u-p)+\left(1-\alpha_{n}\right)\left(x_{n}-\xi_{n} \nabla f\left(x_{n}\right)-p\right)\right\|^{2} \\
\leq & \left(1-\alpha_{n}\right)\left\|x_{n}-\xi_{n} \nabla f\left(x_{n}\right)-p\right\|^{2}+2 \alpha_{n}\left\langle u-p, z_{n}-p\right\rangle \\
\leq & \left(1-\alpha_{n}\right)\left\|x_{n}-p\right\|^{2}+2 \alpha_{n}\left\langle u-p, z_{n}-p\right\rangle \\
& -\left(1-\alpha_{n}\right) \rho_{n}\left(4-\rho_{n}\right) \frac{f^{2}\left(x_{n}\right)}{\left\|\nabla f\left(x_{n}\right)\right\|^{2}} .
\end{aligned}
$$

Therefore, from Lemma 2.6 and (3.2), (3.7) we have

$$
\begin{aligned}
\left\|x_{n+1}-p\right\|^{2}= & \left\|\beta_{n} x_{n}+\left(1-\beta_{n}\right) y_{n}-p\right\|^{2} \\
\leq & \beta_{n}\left\|x_{n}-p\right\|^{2}+\left(1-\beta_{n}\right)\left\|z_{n}-p\right\|^{2} \\
\leq & \beta_{n}\left\|x_{n}-p\right\|^{2}+\left(1-\beta_{n}\right)\left(1-\alpha_{n}\right)\left\|x_{n}-p\right\|^{2}+2 \alpha_{n}\left(1-\beta_{n}\right)\left\langle u-p, z_{n}-p\right\rangle \\
& -\left(1-\alpha_{n}\right)\left(1-\beta_{n}\right) \rho_{n}\left(4-\rho_{n}\right) \frac{f^{2}\left(x_{n}\right)}{\left\|\nabla f\left(x_{n}\right)\right\|^{2}} \\
= & \left\|x_{n}-p\right\|^{2}-\alpha_{n}\left(1-\beta_{n}\right)\left\|x_{n}-p\right\|^{2}+2 \alpha_{n}\left(1-\beta_{n}\right)\left\langle u-p, z_{n}-p\right\rangle \\
& -\left(1-\alpha_{n}\right)\left(1-\beta_{n}\right) \rho_{n}\left(4-\rho_{n}\right) \frac{f^{2}\left(x_{n}\right)}{\left\|\nabla f\left(x_{n}\right)\right\|^{2}} .
\end{aligned}
$$

On the other hand, without loss of generality, we may assume that there is a constant $\sigma>0$ such that

$$
\left(1-\alpha_{n}\right)\left(1-\beta_{n}\right) \rho_{n}\left(4-\rho_{n}\right)>\sigma, \quad \forall n \geq 1 .
$$

Setting $s_{n}=\left\|x_{n}-p\right\|^{2}$, we get the following inequality:

$$
s_{n+1}-s_{n}+\alpha_{n}\left(1-\beta_{n}\right) s_{n}+\frac{\sigma f^{2}\left(x_{n}\right)}{\left\|\nabla f\left(x_{n}\right)\right\|^{2}} \leq 2 \alpha_{n}\left(1-\beta_{n}\right)\left\langle u-p, z_{n}-p\right\rangle .
$$

Now, we prove $s_{n} \rightarrow 0$ by employing the technique studied by Maingé [25]. For the purpose we consider two cases. 
Case 1: $\left\{s_{n}\right\}$ is eventually decreasing, i.e., there exists a sufficient large positive integer $k \geq 1$ such that $s_{n}>s_{n+1}$ holds for all $n \geq k$. In this case, $\left\{s_{n}\right\}$ must be convergent, and from (3.9) it follows that

$$
\frac{\sigma f^{2}\left(x_{n}\right)}{\left\|\nabla f\left(x_{n}\right)\right\|^{2}} \leq\left(s_{n}-s_{n+1}\right)+\alpha_{n}\left(1-\beta_{n}\right) M,
$$

where $M$ is a constant such that $M \geq 2\left\|z_{n}-p\right\|\|u-p\|$ for all $n \in \mathbb{N}$. Using the condition (i) and (3.10), we have

$$
\frac{f^{2}\left(x_{n}\right)}{\left\|\nabla f\left(x_{n}\right)\right\|^{2}} \rightarrow 0 \quad(n \rightarrow \infty)
$$

Moreover, it follows from Lemma 2.1(ii) that for all $n \in \mathbb{N}$

$$
\left\|\nabla f\left(x_{n}\right)\right\|=\left\|\nabla f\left(x_{n}\right)-\nabla f(p)\right\| \leq\|A\|^{2}\left\|x_{n}-p\right\| .
$$

This implies that $\left\{\left\|\nabla f\left(x_{n}\right)\right\|\right\}$ is bounded. From (3.11) it yields $f\left(x_{n}\right) \rightarrow 0$, namely

$$
\left\|(I-T) A x_{n}\right\| \rightarrow 0 .
$$

Furthermore, we have

$$
\lim _{n \rightarrow \infty} \xi_{n}=0 .
$$

For any $x^{*} \in w_{\omega}\left(x_{n}\right)$, and if $\left\{x_{n_{k}}\right\}$ is a subsequence of $\left\{x_{n}\right\}$ such that $x_{n_{k}} \rightarrow x^{*} \in H_{1}$, then

$$
A x_{n_{k}} \rightarrow A x^{*}
$$

On the other hand, from (3.12), we have

$$
\left\|(I-T) A x_{n_{k}}\right\| \rightarrow 0 .
$$

Since $T$ is demi-closed at origin, from (3.14) and (3.15) we have $A x^{*} \in F(T)$, i.e., $A x^{*} \in Q$.

In order to prove $x^{*} \in C=F(S) \cap \Omega$, we need to prove $\lim _{n \rightarrow \infty}\left\|x_{n+1}-x_{n}\right\|=0$ and $\lim _{n \rightarrow \infty}\left\|x_{n}-z_{n}\right\|=0$. In fact, from (3.1) we have

$$
F\left(y_{n}, x\right)+\frac{1}{\lambda_{n}}\left\langle x-y_{n}, y_{n}-z_{n}\right\rangle \geq 0, \quad \forall x \in H_{1} .
$$

Taking $x=y_{n+1}$, we get

$$
F\left(y_{n}, y_{n+1}\right)+\frac{1}{\lambda_{n}}\left\langle y_{n+1}-y_{n}, y_{n}-z_{n}\right\rangle \geq 0 .
$$

Similarly, we also have

$$
F\left(y_{n+1}, y_{n}\right)+\frac{1}{\lambda_{n+1}}\left\langle y_{n}-y_{n+1}, y_{n+1}-z_{n+1}\right\rangle \geq 0 .
$$


Adding up the above two inequalities, we get

$$
F\left(y_{n}, y_{n+1}\right)+F\left(y_{n+1}, y_{n}\right)+\left\langle y_{n+1}-y_{n}, \frac{y_{n}-z_{n}}{\lambda_{n}}-\frac{y_{n+1}-z_{n+1}}{\lambda_{n+1}}\right\rangle \geq 0 .
$$

From (A2), we have

$$
\left\langle y_{n+1}-y_{n}, \frac{y_{n}-z_{n}}{\lambda_{n}}-\frac{y_{n+1}-z_{n+1}}{\lambda_{n+1}}\right\rangle \geq 0 .
$$

Multiplying the above inequality by $\lambda_{n}$ and simplifying, we have

$$
\left\langle y_{n+1}-y_{n}, y_{n}-y_{n+1}+y_{n+1}-z_{n}-\frac{\lambda_{n}}{\lambda_{n+1}}\left(y_{n+1}-z_{n+1}\right)\right\rangle \geq 0 .
$$

Hence we have

$$
\begin{aligned}
\left\|y_{n+1}-y_{n}\right\|^{2} & \leq\left\langle y_{n+1}-y_{n}, y_{n+1}-z_{n}-\frac{\lambda_{n}}{\lambda_{n+1}}\left(y_{n+1}-z_{n+1}\right)\right\rangle \\
& =\left\langle y_{n+1}-y_{n}, z_{n+1}-z_{n}+\left(1-\frac{\lambda_{n}}{\lambda_{n+1}}\right)\left(y_{n+1}-z_{n+1}\right)\right\rangle \\
& \leq\left\|y_{n+1}-y_{n}\right\|\left(\left\|z_{n+1}-z_{n}\right\|+\left|1-\frac{\lambda_{n}}{\lambda_{n+1}}\right| \cdot\left\|y_{n+1}-z_{n+1}\right\|\right)
\end{aligned}
$$

and hence

$$
\begin{aligned}
\left\|y_{n+1}-y_{n}\right\| & \leq\left\|z_{n+1}-z_{n}\right\|+\left|1-\frac{\lambda_{n}}{\lambda_{n+1}}\right|\left\|y_{n+1}-z_{n+1}\right\| \\
& \leq\left\|z_{n+1}-z_{n}\right\|+\frac{1}{a}\left|\lambda_{n+1}-\lambda_{n}\right| \cdot\left\|y_{n+1}-z_{n+1}\right\| .
\end{aligned}
$$

By (3.1) we have

$$
\begin{aligned}
\left\|z_{n+1}-z_{n}\right\|= & \| S\left(\alpha_{n+1} u+\left(1-\alpha_{n+1}\right)\left(x_{n+1}-\xi_{n+1} \nabla f\left(x_{n+1}\right)\right)\right) \\
& -S\left(\alpha_{n} u+\left(1-\alpha_{n}\right)\left(x_{n}-\xi_{n} \nabla f\left(x_{n}\right)\right)\right) \| \\
\leq & \|\left(\alpha_{n+1}-\alpha_{n}\right) u \\
& +\left(1-\alpha_{n+1}\right)\left\{\left(x_{n+1}-\xi_{n+1} \nabla f\left(x_{n+1}\right)\right)-\left(x_{n}-\xi_{n} \nabla f\left(x_{n}\right)\right)\right\} \\
& -\left(\alpha_{n+1}-\alpha_{n}\right)\left(x_{n}-\xi_{n} \nabla f\left(x_{n}\right)\right) \| \\
\leq & \left(1-\alpha_{n+1}\right)\left\|x_{n+1}-x_{n}\right\|+N_{n} \leq\left\|x_{n+1}-x_{n}\right\|+N_{n},
\end{aligned}
$$

where

$$
\begin{aligned}
N_{n}= & \left|\alpha_{n+1}-\alpha_{n}\right| \cdot\|u\|+\left(1-\alpha_{n+1}\right)\left(\xi_{n+1}\left\|\nabla f\left(x_{n+1}\right)\right\|+\xi_{n}\left\|\nabla f\left(x_{n}\right)\right\|\right) \\
& +\left|\alpha_{n+1}-\alpha_{n}\right| \cdot\left\|x_{n}-\xi_{n} \nabla f\left(x_{n}\right)\right\| \rightarrow 0 \quad(n \rightarrow \infty) .
\end{aligned}
$$

This implies that

$$
\left\|y_{n+1}-y_{n}\right\| \leq\left\|x_{n+1}-x_{n}\right\|+\frac{1}{a}\left|\lambda_{n+1}-\lambda_{n}\right| \cdot\left\|y_{n+1}-z_{n+1}\right\|+N_{n} .
$$


It follows that

$$
\left\|y_{n+1}-y_{n}\right\|-\left\|x_{n+1}-x_{n}\right\| \leq \frac{1}{a}\left|\lambda_{n+1}-\lambda_{n}\right| \cdot\left\|y_{n+1}-z_{n+1}\right\|+N_{n} .
$$

In view of condition (iii) and (3.17) we get

$$
\limsup _{n \rightarrow \infty}\left(\left\|y_{n+1}-y_{n}\right\|-\left\|x_{n+1}-x_{n}\right\|\right) \leq 0
$$

By Lemma 2.5, we obtain

$$
\lim _{n \rightarrow \infty}\left\|y_{n}-x_{n}\right\|=0
$$

Consequently

$$
\begin{aligned}
\left\|x_{n+1}-x_{n}\right\| & =\left\|\beta_{n} x_{n}+\left(1-\beta_{n}\right) y_{n}-x_{n}\right\| \\
& =\left(1-\beta_{n}\right)\left\|y_{n}-x_{n}\right\| \rightarrow 0 \quad(n \rightarrow \infty) .
\end{aligned}
$$

Since $S$ is firmly nonexpansive, it follows from (3.1) that

$$
\begin{aligned}
2\left\|z_{n}-p\right\|^{2}= & 2\left\|S\left(\alpha_{n} u+\left(1-\alpha_{n}\right)\left(x_{n}-\xi_{n} \nabla f\left(x_{n}\right)\right)\right)-S p\right\|^{2} \\
\leq & 2\left\langle\alpha_{n} u+\left(1-\alpha_{n}\right)\left(x_{n}-\xi_{n} \nabla f\left(x_{n}\right)\right)-p, z_{n}-p\right\rangle \\
= & \left\|\alpha_{n} u+\left(1-\alpha_{n}\right)\left(x_{n}-\xi_{n} \nabla f\left(x_{n}\right)\right)-p\right\|^{2}+\left\|z_{n}-p\right\|^{2} \\
& -\left\|\alpha_{n} u+\left(1-\alpha_{n}\right)\left(x_{n}-\xi_{n} \nabla f\left(x_{n}\right)\right)-p-z_{n}+p\right\|^{2} \\
= & \left\|\alpha_{n}(u-p)+\left(1-\alpha_{n}\right)\left(x_{n}-\xi_{n} \nabla f\left(x_{n}\right)-p\right)\right\|^{2}+\left\|z_{n}-p\right\|^{2} \\
& -\left\|\alpha_{n}\left(u-z_{n}\right)+\left(1-\alpha_{n}\right)\left(x_{n}-\xi_{n} \nabla f\left(x_{n}\right)-z_{n}\right)\right\|^{2} \\
\leq & \left(1-\alpha_{n}\right)\left\|x_{n}-p\right\|^{2}+\left\|z_{n}-p\right\|^{2}-\left\|x_{n}-z_{n}\right\|^{2}+M_{n},
\end{aligned}
$$

where

$$
\begin{aligned}
M_{n}:= & \alpha_{n}\|u-p\|^{2}+\left(1-\alpha_{n}\right)\left\|\xi_{n} \nabla f\left(x_{n}\right)\right\|^{2}-2\left(1-\alpha_{n}\right) \xi_{n}\left\langle x_{n}-p, \nabla f\left(x_{n}\right)\right\rangle \\
& -\alpha_{n}\left\|u-z_{n}\right\|^{2}-\left(1-\alpha_{n}\right)\left\{\left\|\xi_{n} \nabla f\left(x_{n}\right)\right\|^{2}-2\left\langle x_{n}-z_{n}, \xi_{n} \nabla f\left(x_{n}\right)\right\rangle\right\} \\
& +\alpha_{n}\left\|x_{n}-z_{n}\right\|^{2}+\alpha_{n}\left(1-\alpha_{n}\right)\left\|x_{n}-u-\xi_{n} \nabla f\left(x_{n}\right)\right\|^{2} \\
\rightarrow & 0 \quad(\operatorname{as} n \rightarrow \infty) .
\end{aligned}
$$

Therefore we have

$$
\left\|z_{n}-p\right\|^{2} \leq\left\|x_{n}-p\right\|^{2}-\left\|x_{n}-z_{n}\right\|^{2}+M_{n} .
$$

This together with (3.8) shows that

$$
\begin{aligned}
\left\|x_{n+1}-p\right\|^{2} & \leq \beta_{n}\left\|x_{n}-p\right\|^{2}+\left(1-\beta_{n}\right)\left\|z_{n}-p\right\|^{2} \\
& \leq\left\|x_{n}-p\right\|^{2}-\left(1-\beta_{n}\right)\left\|x_{n}-z_{n}\right\|^{2}+\left(1-\beta_{n}\right) M_{n} .
\end{aligned}
$$


Then we obtain

$$
\begin{aligned}
\left(1-\beta_{n}\right)\left\|x_{n}-z_{n}\right\|^{2} & \leq\left\|x_{n}-p\right\|^{2}-\left\|x_{n+1}-p\right\|^{2}+\left(1-\beta_{n}\right) M_{n} \\
& =s_{n}-s_{n+1}+\left(1-\beta_{n}\right) M_{n} .
\end{aligned}
$$

Therefore, we get

$$
\lim _{n \rightarrow \infty}\left\|x_{n}-z_{n}\right\|=0
$$

By virtue of (3.18), we have

$$
\lim _{n \rightarrow \infty}\left\|y_{n}-z_{n}\right\|=0
$$

Now, we turn to a proof that $x^{*} \in C=F(S) \cap \Omega$. For this purpose, we denote

$$
v_{n}:=\alpha_{n} u+\left(1-\alpha_{n}\right)\left(x_{n}-\xi_{n} \nabla f\left(x_{n}\right)\right) .
$$

In view of condition (i) and (3.13) we have

$$
\begin{aligned}
\left\|v_{n}-x_{n}\right\| & =\left\|\alpha_{n} u+\left(1-\alpha_{n}\right)\left(x_{n}-\xi_{n} \nabla f\left(x_{n}\right)\right)-x_{n}\right\| \\
& =\left\|\alpha_{n}\left(u-x_{n}\right)-\left(1-\alpha_{n}\right) \xi_{n} \nabla f\left(x_{n}\right)\right\| \\
& \leq \alpha_{n}\left\|u-x_{n}\right\|+\left(1-\alpha_{n}\right) \xi_{n}\left\|\nabla f\left(x_{n}\right)\right\| \rightarrow 0 .
\end{aligned}
$$

Since $S$ is firmly nonexpansive (and so it is also nonexpansive), it follows from Lemma 2.4 that

$$
\begin{aligned}
\left\|z_{n+1}-p\right\|^{2} & =\left\|S v_{n+1}-S x_{n}+S x_{n}-S p\right\|^{2} \\
& \leq\left\|S x_{n}-S p\right\|^{2}+2\left\langle S v_{n+1}-S x_{n+1}+S x_{n+1}-S x_{n}, z_{n+1}-p\right\rangle \\
& \leq\left\|x_{n}-p\right\|^{2}-\left\|(I-S) x_{n}\right\|^{2}+2\left(\left\|v_{n+1}-x_{n+1}\right\|+\left\|x_{n+1}-x_{n}\right\|\right)\left\|z_{n+1}-p\right\| .
\end{aligned}
$$

Thus, we have

$$
\begin{aligned}
&\left\|(I-S) x_{n}\right\|^{2} \\
& \leq\left\|x_{n}-p\right\|^{2}-\left\|z_{n+1}-p\right\|^{2}+2\left(\left\|v_{n+1}-x_{n+1}\right\|+\left\|x_{n+1}-x_{n}\right\|\right)\left\|z_{n+1}-p\right\| \\
& \leq\left\|x_{n}-p\right\|^{2}-\left(\left\|z_{n+1}-x_{n+1}\right\|-\left\|x_{n+1}-p\right\|\right)^{2} \\
&+2\left(\left\|v_{n+1}-x_{n+1}\right\|+\left\|x_{n+1}-x_{n}\right\|\right)\left\|z_{n+1}-p\right\| \\
& \leq\left\|x_{n}-p\right\|^{2}-\left\|x_{n+1}-p\right\|^{2}-\left\|z_{n+1}-x_{n+1}\right\|^{2}+2\left\|x_{n+1}-p\right\| \cdot\left\|z_{n+1}-x_{n+1}\right\| \\
&+2\left(\left\|v_{n+1}-x_{n+1}\right\|+\left\|x_{n+1}-x_{n}\right\|\right)\left\|z_{n+1}-p\right\| \\
&= s_{n}-s_{n+1}-\left\|z_{n+1}-x_{n+1}\right\|^{2}+2\left\|x_{n+1}-p\right\| \cdot\left\|z_{n+1}-x_{n+1}\right\| \\
&+2\left(\left\|v_{n+1}-x_{n+1}\right\|+\left\|x_{n+1}-x_{n}\right\|\right)\left\|z_{n+1}-p\right\| .
\end{aligned}
$$

It follows from (3.19), (3.20), and (3.22) that $\left\|(I-S) x_{n}\right\| \rightarrow 0$. In view of $x_{n_{k}} \rightarrow x^{*}$ and that $S$ is demi-closed at origin, we get $x^{*} \in F(S)$. 
On the other hand, from $x_{n_{k}} \rightarrow x^{*}$ and (3.18), we obtain $y_{n_{k}} \rightarrow x^{*}$. From (3.1), for any $x \in H_{1}$, we have

$$
F\left(y_{n}, x\right)+\frac{1}{\lambda_{n}}\left\langle x-y_{n}, y_{n}-z_{n}\right\rangle \geq 0 .
$$

From (A2), we have

$$
\frac{1}{\lambda_{n}}\left\langle x-y_{n}, y_{n}-z_{n}\right\rangle \geq F\left(x, y_{n}\right), \quad \forall x \in H_{1} .
$$

Replacing $n$ by $n_{k}$, we have

$$
\left\langle x-y_{n_{k}}, \frac{y_{n_{k}}-z_{n_{k}}}{\lambda_{n_{k}}}\right\rangle \geq F\left(x, y_{n_{k}}\right), \quad \forall x \in H_{1} .
$$

Since $\left\|\frac{y_{n_{k}}-z_{n_{k}}}{\lambda_{n_{k}}}\right\| \rightarrow 0$ and $y_{n_{k}} \rightarrow x^{*}$, from (A4) we have

$$
F\left(x, x^{*}\right) \leq 0, \quad \forall x \in H_{1} .
$$

Put $w_{t}=t x+(1-t) x^{*}$ for all $t \in(0,1]$ and $x \in H_{1}$. Then we get $w_{t} \in H_{1}$. So, from (3.24) we have

$$
F\left(w_{t}, x^{*}\right) \leq 0, \quad \forall x \in H_{1} .
$$

From (A4), we have

$$
\begin{aligned}
0=F\left(w_{t}, w_{t}\right) & \leq t F\left(w_{t}, x\right)+(1-t) F\left(w_{t}, x^{*}\right) \\
& \leq t F\left(w_{t}, x\right),
\end{aligned}
$$

and hence $F\left(w_{t}, x\right) \geq 0$. Letting $t \rightarrow 0$, we have

$$
F\left(x^{*}, x\right) \geq 0, \quad \forall x \in H_{1} .
$$

This implies $x^{*} \in \Omega$. Consequently, $x^{*} \in C$, and hence $w_{w}\left(x_{n}\right) \subset \Gamma$. Furthermore, in view of (3.20) we have

$$
\begin{aligned}
\limsup _{n \rightarrow \infty}\left\langle u-p, z_{n}-p\right\rangle & =\limsup _{n \rightarrow \infty}\left\langle u-p, x_{n}-p\right\rangle \\
& =\max _{w \in w_{w}\left(x_{n}\right)}\left\langle u-P_{\Gamma} u, w-P_{\Gamma} u\right\rangle \leq 0 .
\end{aligned}
$$

On the other hand, from (3.9), we have

$$
s_{n+1} \leq\left(1-\alpha_{n}\left(1-\beta_{n}\right)\right) s_{n}+2 \alpha_{n}\left(1-\beta_{n}\right)\left\langle u-p, z_{n}-p\right\rangle .
$$

Applying Lemma 2.6 to (3.25), from the condition (i) we obtain $s_{n} \rightarrow 0$, that is, $x_{n} \rightarrow p$. 
Case 2: $\left\{s_{n}\right\}$ is not eventually decreasing, that is, we can find a positive integer $n_{0}$ such that $s_{n_{0}} \leq s_{n_{0}+1}$. Now we define

$$
U_{n}:=\left\{n_{0} \leq k \leq n: s_{k} \leq s_{k+1}\right\}, \quad n>n_{0} .
$$

It easy to see that $U_{n}$ is nonempty and satisfies $U_{n} \subseteq U_{n+1}$. Let

$$
\psi(n):=\max U_{n}, \quad n>n_{0}
$$

It is clear that $\psi(n) \rightarrow \infty$ as $n \rightarrow \infty$ (otherwise, $\left\{s_{n}\right\}$ is eventually decreasing). It is also clear that $s_{\psi(n)} \leq s_{\psi(n)+1}$ for all $n>n_{0}$. Moreover, we prove that

$$
s_{n} \leq s_{\psi(n)+1}, \quad \forall n>n_{0} .
$$

In fact, if $\psi(n)=n$, then the inequality (3.26) is trivial; if $\psi(n)<n$, from the definition of $\psi(n)$, there exists some $i \in \mathbb{N}$ such that $\psi(n)+i=n$, we deduce that

$$
s_{\psi(n)+1}>s_{\psi(n)+2}>\cdots>s_{\psi(n)+i}=s_{n}
$$

and the inequality (3.26) holds again. Since $s_{\psi(n)} \leq s_{\psi(n)+1}$ for all $n>n_{0}$, it follows from (3.10) that

$$
\frac{\sigma f^{2}\left(x_{\psi(n)}\right)}{\left\|\nabla f\left(x_{\psi(n)}\right)\right\|^{2}} \leq \alpha_{\psi(n)}\left(1-\beta_{\psi(n)}\right) M \rightarrow 0 .
$$

Noting that $\left\{\left\|\nabla f\left(x_{\psi(n)}\right)\right\|\right\}$ is bounded, we get $f\left(x_{\psi(n)}\right) \rightarrow 0$. By the same argument to the proof in case 1 , we have $w_{w}\left(x_{\psi(n)}\right) \subset \Gamma$. From (3.19) we have

$$
\lim _{n \rightarrow \infty}\left\|x_{\psi(n)}-x_{\psi(n)+1}\right\|=0
$$

Furthermore, in view of (3.20), we can deduce that

$$
\begin{aligned}
& \limsup _{n \rightarrow \infty}\left\langle u-p, z_{\psi(n)}-p\right\rangle \\
& \quad=\limsup _{n \rightarrow \infty}\left\langle u-p, x_{\psi(n)}-p\right\rangle \\
& \quad=\max _{w \in w_{w}\left(x_{\psi(n)}\right)}\left\langle u-P_{\Gamma} u, w-P_{\Gamma} u\right\rangle \leq 0 .
\end{aligned}
$$

Since $s_{\psi(n)} \leq s_{\psi(n)+1}$, it follows from (3.9) that

$$
s_{\psi(n)} \leq 2\left\langle u-p, z_{\psi(n)}-p\right\rangle, \quad n>n_{0} .
$$

Combining (3.28) and (3.29) we have

$$
\limsup _{n \rightarrow \infty} s_{\psi(n)} \leq 0
$$


and hence $s_{\psi(n)} \rightarrow 0$, which together with (3.27) implies that

$$
\begin{aligned}
\sqrt{s_{\psi(n)+1}} & \leq\left\|\left(x_{\psi(n)}-p\right)+\left(x_{\psi(n)+1}-x_{\psi(n)}\right)\right\| \\
& \leq \sqrt{s_{\psi(n)}}+\left\|x_{\psi(n)+1}-x_{\psi(n)}\right\| \rightarrow 0 .
\end{aligned}
$$

Noting the inequality (3.26), this shows that $s_{n} \rightarrow 0$, that is, $x_{n} \rightarrow p$. This completes the proof of Theorem 3.1.

\section{Competing interests}

The authors declare that they have no competing interests.

\section{Authors' contributions}

All authors contributed equally and significantly to this research work. All authors read and approved the final manuscript.

\section{Author details}

'Department of Mathematics, Yibin University, Yibin, Sichuan 644007, China. ${ }^{2}$ College of Statistics and Mathematics, Yunnan University of Finance and Economics, Kunming, Yunnan 650221, China. ${ }^{3}$ Faculty of Computing, Engineering and Technology, Staffordshire University, Beaconside, Stafford, Staffordshire ST18 0AD, UK.

\section{Acknowledgements}

This study was supported by the Scientific Research Fund of Sichuan Provincial Education Department (13ZA0199) and the Scientific Research Fund of Sichuan Provincial Department of Science and Technology (2012JYZ011) and by the National Natural Science Foundation of China (Grant No. 11361070).

Received: 16 June 2013 Accepted: 31 January 2014 Published: 13 Feb 2014

\section{References}

1. Blum, $\mathrm{E}$, Oettli, W: From optimization and variational inequalities to equilibrium problems. Math. Stud. $63,123-145$ (1994)

2. Chadli, O, Wong, NC, Yao, JC: Equilibrium problems with applications to eigenvalue problems. J. Optim. Theory Appl. $117(2), 245-266(2003)$

3. Chadli, O, Schaible, S, Yao, JC: Regularized equilibrium problems with an application to noncoercive hemivariational inequalities. J. Optim. Theory Appl. 121, 571-596 (2004)

4. Combettes, PL, Hirstoaga, SA: Equilibrium programming in Hilbert space. J. Nonlinear Convex Anal. 6, 117-136 (2005)

5. Ceng, LC, Yao, JC: A hybrid iterative scheme for mixed equilibrium problems and fixed point problems. J. Comput. Appl. Math. 214, 186-201 (2008)

6. Takahashi, S, Takahashi, W: Strong convergence theorem for a generalized equilibrium problem and a nonexpansive mapping in a Hilbert space. Nonlinear Anal. 69, 1025-1033 (2008)

7. Reich, S, Sabach, S: Three strong convergence theorems regarding iterative methods for solving equilibrium problems in reflexive Banach spaces, optimization theory and related topics. Contemp. Math. 568, 225-240 (2012)

8. Kassay, G, Reich, S, Sabach, S: Iterative methods for solving systems of variational inequalities in reflexive Banach spaces. SIAM J. Optim. 21, 1319-1344 (2011)

9. Censor, Y, Elfving, T: A multiprojection algorithm using Bregman projection in product space. Numer. Algorithms 8 , 221-239 (1994)

10. Aleyner, A, Reich, S: Block-iterative algorithms for solving convex feasibility problems in Hilbert and in Banach J. Math. Anal. Appl. 343(1), 427-435 (2008)

11. Bauschke, HH, Borwein, JM: On projection algorithms for solving convex feasibility problems. SIAM Rev. 38(3), 367-426 (1996)

12. Moudafi, A: A relaxed alternating CQ-algorithm for convex feasibility problems. Nonlinear Anal. 79, 117-121 (2013)

13. Masad, E, Reich, S: A note on the multiple-set split convex feasibility problem in Hilbert space. J. Nonlinear Convex Anal. 8, 367-371 (2007)

14. Yao, Y, Chen, R, Marino, G, Liou, YC: Applications of fixed point and optimization methods to the multiple-sets split feasibility problem. J. Appl. Math. 2012, Article ID 927530 (2012)

15. Xu, HK: A variable Krasnosel'skii-Mann algorithm and the multiple-set split feasibility problem. Inverse Probl. 22, 2021-2034 (2006)

16. Xu, HK: Iterative methods for the split feasibility problem in infinite-dimensional Hilbert spaces. Inverse Probl. 26(10), Article ID 105018 (2010)

17. Yang, Q: The relaxed CQ algorithm for solving the split feasibility problem. Inverse Probl. 20, 1261-1266 (2004)

18. Zhao, J, Yang, Q: Several solution methods for the split feasibility problem. Inverse Probl. 21, 1791-1799 (2005)

19. López, G, Martín-Márquez, V, Wang, FH, Xu, HK: Solving the split feasibility problem without prior knowledge of matrix norms. Inverse Probl. 28, 085004 (2012). doi:10.1088/0266-5611/28/8/085004

20. He, S, Zhao, Z: Strong convergence of a relaxed CQ algorithm for the split feasibility problem. J. Inequal. Appl. 2013, 197 (2013). doi:10.1186/1029-242X-2013-197

21. Goebel, K, Reich, S: Uniform Convexity, Hyperbolic Geometry and Nonexpansive Mappings. Marcel Dekker, New York (1984) 
22. Chang, SS: On Chidume's open questions and approximate solutions for multi-valued strongly accretive mapping equations in Banach spaces. J. Math. Anal. Appl. 216, 94-111 (1997)

23. Suzuki, T: Strong convergence of Krasnoselskii and Mann's type sequences for one-parameter nonexpansive semigroups without Bochner integrals. J. Math. Anal. Appl. 305, 227-239 (2005)

24. Xu, HK: Iterative algorithms for nonlinear operators. J. Lond. Math. Soc. 66, 240-256 (2002)

25. Maingé, PE: New approach to solving a system of variational inequalities and hierarchical problems. J. Optim. Theory Appl. 138, 459-477 (2008)

10.1186/1687-1812-2014-36

Cite this article as: Tang et al.: A strong convergence theorem for equilibrium problems and split feasibility problems in Hilbert spaces. Fixed Point Theory and Applications 2014, 2014:36

\section{Submit your manuscript to a SpringerOpen ${ }^{\circ}$ journal and benefit from:}

- Convenient online submission

- Rigorous peer review

- Immediate publication on acceptance

Open access: articles freely available online

- High visibility within the field

- Retaining the copyright to your article 